\title{
An Application of Linear Automata to Near Rings
}

\author{
Songfa You, Yijun Feng, Ming Cao, Yaping Wei \\ School of Mathematics and Computer Science, Hubei University, Wuhan, China \\ Email: yousongfa@163.com
}

Received August 31, 2012; revised September 30, 2012; accepted October 7, 2012

\begin{abstract}
In this paper, we have established an intimate connection between near-nings and linear automata, and obtain the following results: 1) For a near-ring $N$ there exists a linear GSA $S$ with $N \cong N(\boldsymbol{S})$ iff (a) $(N,+)$ is abelian, (b) $N$ has an identity 1, (c) There is some $d \in N_{d}$ such that $N_{0}$ is generated by $\{1, d\}$; 2) Let $h: S \rightarrow \boldsymbol{S}^{\prime}$ be a GSAepimorphism. Then there exists a near-ring epimorphism $\bar{h}$ from $N(\boldsymbol{S})$ to $N\left(\boldsymbol{S}^{\prime}\right)$ with $h(q n)=h(q) \bar{h}(n)$ for all $q \in Q$ and $n \in N(\boldsymbol{S})$; 3) Let $\boldsymbol{A}=(Q, A, B, F, G)$ be $a$ GA. Then (a) $\boldsymbol{A}_{a}:=\left(Q(N(\boldsymbol{A}))=: Q_{a}, A, B, F / Q_{a} \times A, G / Q_{a} \times A\right)$ is accessible, (b) $Q=0 N(A)$, (c) $A / \sim:=\left(Q / \sim, A, B, F_{\sim}, Q_{\sim}\right)$ with $F_{\sim}([q], a):=[F(q, a)]$ and $G_{\sim}([q], a):=G(q, a)$ is reduced, (d) $A_{a} / \sim$ is minimal.
\end{abstract}

Keywords: Linear Automata; Accessible; GSA-Homomorphism; Near-Ring

\section{Introduction}

Automata consist of inputs, states, and outputs, together with maps which describe how new inputs affect the state and the output. A semi-automation is a triple

$\boldsymbol{S}=(Q, A, F)$, where $Q$ and $A$ are sets, called the state set and input set, and $F$ is a function from $Q \times A$ in $Q$, called the state-transition function. If $Q$ is a group, we call $S$ a group-semiautomaton and abbreviate this by GSA. Automata consist of inputs, states, and outputs, together with maps which describe how new inputs affect the state and the output. A semiautomaton is a triple $\boldsymbol{S}=(Q, A, F)$, where $Q$ and $A$ are sets, called the state set and the input set, and $F$ is a function from $Q \times A$ in $Q$, called the state-transition function. If $Q$ is a group (we always write it additively), we call $S$ a group-semiautomaton and abbreviate this by GSA. For $q \in Q$ and $a \in A$ we interprete $F(q, a)$ as the new state obtained from the old state $q$ by mean of the input $a$ [1].

If $\boldsymbol{S}=(Q, A, F)$ is a semiautomaton, we get a collection of mappings $f_{a}$ from $Q$ to $Q$, one for each $a \in A$, which are given by $q f_{a}:=F(q, a)$. Hence $f_{a}$ describes the effect of the input $a$ on the state set $Q$ of $S$.

If the input $a_{1} \in A$ is followed by the input $a_{2}$, the semiautomaton moves from the state $q \in Q$ first into $q f_{a_{1}}$ and then into $\left(q f_{a_{1}}\right) f_{a_{2}}$. We extend (as usual) $A$ to the free monoid $A^{*}$ over $A$ consisting of all finite sequences of elements of $A$, including the empty sequence $\wedge$, and get $f_{a_{1} a_{2}}=f_{a_{1}} f_{a_{2}}$, i.e. the map $a \rightarrow f_{a}$ is a monomorphism from $A^{*}$ into the transformation monoid over $Q$ with $f_{\wedge}=i d_{Q}$. In the case of GSA's, we are also able to study the superposition $f_{a_{1}}+f_{a_{2}}$ (defined pointwisely) of two simultaneous inputs $a_{1}, a_{2} \in A$. Hence it is natural to consider $\left\{f_{a} \mid a \in A\right\} \cup\left\{f_{\wedge}\right\}$ and all of its sums and products (composition of maps). The obvious framework for that is, of course, the structure of a near ring.

Let $\boldsymbol{S}=(Q, A, F)$ be a $G S A$, The subnear-ring $N(\boldsymbol{S})$ of $M(Q)$ generated by $i d_{Q}$ and all $f_{\alpha}^{\prime} s$ is called the syntactic near-ring of $\boldsymbol{S}$. Thus $N(\boldsymbol{S})$ is always a near-ring with identity. If $Q$ is finite, then $N(\boldsymbol{S})$ is finite, too [2].

\section{Discussion}

1) The homomorphism case. Let $Q$ and A be additive groups with zero 0 and $F a$ homomorphism from the direct product $Q \times A$. We then call $(Q, A, F)$ a homomorphic $G S A$. Because of $q f_{a}=F(q, a)=F(q, 0)+$ $(0, a)=F(q, 0)+F(0, a)=q f_{0}+o f_{a}$, we get $f_{a}=f_{0}$ $+\bar{f}_{a}$, where $f_{0}$ is a homomorphism (i.e. a distributive element in $N(Q)$ ), while $\bar{f}_{a}$ is the map with constant value $0 f_{a}$. If no input can change the zero state, i.e. if $0 f_{a}=0$ for all $a \in A$, then $N(\boldsymbol{S})$ obviously is $a$ distributively generated near-ring, consisting of \pm -sums of powers of $f_{0}$ which are endomorphisms, we also get $a$ distributively generated near-ring if $F$ is additive in the first component. For homomorphic GSA's one sees by 
induction that

$f_{a_{1} a_{2} \cdots a_{n}}=f_{0}^{n}+\left(\bar{f}_{a_{1}} f_{0}^{n-1}+\cdots+\bar{f}_{a_{n-1}} f_{0}+\bar{f}_{a_{n}}\right)$, where the map in brackets is constant. Each power $f_{0}^{n}$ is a homomorphism [3].

2) The linear case is a special case of the homomorphism case in which $Q$ and $A$ are Abelian groups (or more generally, R-modules for some ring $R$ ) and where $F$ is linear. Let $Q$ and $A$ be free R-modules with finite base $X$, $Y$ respectively. Let $|X|=n,|Y|=m$. Then the action of $F$ can be described by an $m \times(n+m)$-matrix $Z=\left(z_{i j}\right)$ over $R$ if we replace each element of $Q$ and of $A$ by its decomposition $f_{a}=f_{0}+\bar{f}_{a}$ induces a decomposition of $\mathrm{Z}$ such that

$$
\begin{aligned}
F(q, a) & =Z \cdot(q, a) \\
& =\left(\begin{array}{ccc}
z_{11} & \cdots & z_{1 m} \\
\vdots & \ddots & \vdots \\
z_{m 1} & \cdots & z_{m m}
\end{array}\right) \cdot q+\left(\begin{array}{ccc}
z_{1 m+1} & \cdots & z_{1 m+n} \\
\vdots & \ddots & \vdots \\
z_{m, m+1} & \cdots & z_{m, m+n}
\end{array}\right) \cdot a \\
& =: B \cdot q+C \cdot a
\end{aligned}
$$

We then get

$q f_{a_{1} a_{2} \cdots a_{k}}=B^{k} \cdot q+B^{k-1} \cdot C \cdot a_{1}+\cdots+B \cdot C \cdot a_{k-1}+C \cdot a_{k}$. If, in particular, $C=0$, we get $q f_{a_{1} \cdots a_{k}}=B^{k} \cdot q$ and $N(\boldsymbol{S})$ is a ring, generated by $B$ and the unit matrix I [4]. on the other hand, if $B=0$, then $q f_{a_{1} \cdots a_{k}}=C \cdot a_{k}$. We get $f_{a_{1} \cdots a_{k}}=f_{a_{1}^{\prime} \cdots a_{k}^{\prime}}$ iff $C \cdot\left(a_{k}-a_{k}^{\prime}\right)=0$.

Anyhow, each $f_{a}$ (and hence each $f_{a}$ for $a \in A^{*}$ ) is an affine map from $Q$ to $Q$. If $Q$ is free on $X$ with $|X|=n$ then we can extend the idea of matrix representations from linear maps to affine maps. Let $f$ be an affine map. Then $f$ decomposes as $f=f_{0}+c$ where $f_{0}$ is a homomorphism and $c$ is constant. Let $F$ be the matrix for $f_{0}$ with respect to $X$. Invent a symbol $e$ with $e+e=e e=e$ and $e r=r e=e$ for all $r \in R$. Then

$$
f \rightarrow\left(\begin{array}{cc}
F & 0 \\
c^{t} & e
\end{array}\right)
$$

Establishes an isomorphism between $M_{\text {aff }}(Q)$ (all affine of $Q)$ and a subnear-ring of all $(n+1) \times(n+1)$ matrices over $R \bigcup\{e\}$ [3].

\section{Main Results}

Theorem 1. Let $S=(Q, A, F)$ be a homomorphic GSA, Then $N(\boldsymbol{S})=\left\{\sum \pm f_{\alpha_{i}} \mid \alpha_{i} \in A^{*}\right\}=: N$

Proof. $N \subseteq N(\boldsymbol{S})$ is clear. Conversely it suffices to show that $N$ is a near-ring, since obviously $N$ contains all $f_{a}(a \in A)$ and $i d_{Q}=f$. In fact, we show that $N$ is a subnear-ring of $M(Q)$

Take $f=\sum_{i} \pm f_{\alpha_{i}} \in N, g=\sum_{j} \pm f_{\beta_{j}} \in N$. It is clear that $f+g \in N$. So consider

$$
f g: f g=\left(\sum_{i} \pm f_{\alpha_{i}}\right)\left(\sum_{j} \pm f_{\beta_{j}}\right)=\sum_{j} \pm\left(\sum_{i} \pm f_{\alpha_{i}}\right) f_{\beta_{j}} .
$$

Hence we only look at the last expression in (a), let $\beta_{j}=a_{1} a_{2} \cdots a_{n} \in A^{*}$. Then

$$
\left(\sum_{i} \pm f_{\alpha_{i}}\right) f_{\beta_{j}}=\left(\sum_{i} \pm f_{\alpha_{j}}\right) f_{a_{1}} f_{a_{2}} \cdots f_{a_{n}}
$$

We first focus our attention to $n=1$ and put $a_{1}=a$ for a moment

$$
\begin{aligned}
\left(\sum_{i} \pm f_{\alpha_{i}}\right) f_{a} & =\left(\sum_{i} \pm f_{\alpha_{i}}\right) f_{0}+\bar{f}_{a} \\
& =\left(\sum_{i} \pm f_{\alpha_{i}} f_{0}\right)+\bar{f}_{a}=\left(\sum_{i} \pm f_{\alpha_{i} 0}\right)+\bar{f}_{a} \\
& =\left(\sum_{i} \pm f_{\alpha_{i} 0}\right)-f_{0}+f_{a} \in N
\end{aligned}
$$

Therefore we get $\gamma_{k} \in A^{*}$ with

$$
\begin{aligned}
\left(\sum_{i} \pm f_{\alpha_{i}}\right) f_{a_{1}} f_{a_{2}} \cdots f_{a_{n}} & =\left(\left(\sum \pm f_{\alpha_{i}}\right) f_{a_{1}}\right) f_{a_{2}} \cdots f_{a_{n}} \\
& =\left(\sum_{k} \pm f_{\gamma_{k}}\right) f_{a_{2}} \cdots f_{a_{n}}
\end{aligned}
$$

By induction, this is in $N$

Let $\boldsymbol{S}=(Q, A, F)$ be homomorphic. The zero-symmetric part $N_{0}(\boldsymbol{S}):=(N(\boldsymbol{S}))_{0}$, and $N_{0}(\boldsymbol{S})$ consists of all finite sums of elements of the form $c \pm f-c$ with $f \in\left\{i d, f_{0}, f_{0}^{2}, f_{0}^{3}, \cdots\right\}$ and $c \in\left\{\sum \pm \bar{f}_{\alpha_{i}} \mid \alpha_{i} \in A^{*}\right\}$.

In fact, all elements $c \pm f-c$ are in $N_{0}(\boldsymbol{S})$. Conversely, take $\left.g=\Sigma \pm f_{\alpha_{i}} \in N_{0}(S)\right\}$. Then

$0=0 g=0\left(\Sigma \pm f_{\alpha_{i}}\right)=\Sigma \pm 0 f_{\alpha_{i}}=\Sigma \pm \bar{f}_{\alpha_{i}}$. By standard group theory, we can arrange

$g=\Sigma \pm f_{\alpha_{i}}=\Sigma \pm\left(f_{0}^{n_{i}}+\bar{f}_{\alpha_{i}}\right)$ into sums and differences of elements of the form $c+f_{0}^{n_{i}}-c$, where $c$ is the sum of some $\bar{f}_{\alpha_{i}}^{\prime} s$ [5]. If $\boldsymbol{S}$ be linear. Then (with $f_{0}^{0}:=i d$ ) $N_{0}(\boldsymbol{S})=\left\{z_{0} f_{0}^{0}+z_{1} f_{0}^{1}+\cdots+z_{n} f_{0}^{n} \mid z_{i} \in Z\right\} \quad(n$ is non negative integer ), Hence $N_{0}(S)$ is the subnear-ring of $M_{\text {aff }}(Q)$ generated by $\left\{i d, f_{0}\right\}$. Since $\left(M_{\text {aff }}(Q)\right)_{0}$ is $a$ ring, $N_{0}(\boldsymbol{S})$ is a ring, too [6].

We can find a group $Q$ such that $N$ is isomorphic to a subnear-ring $\bar{N}$ of $M(Q)$. Let $A$ be an index set for $\bar{N}$, i.e. $\bar{N}=\left\{f_{a} \mid a \in A\right\}$. Let $F(q, a):=q f_{a}$. Then $N \cong \bar{N}=N(\boldsymbol{S})$ with $\boldsymbol{S}=(Q, A, F)$. Since every nearring can be embedded in a near-ring with identity, we get every near-ring can be embedded in the near-ring of some GSA [7]

Theorem 2. For a near-ring $N$ there exists a linear GSA $\boldsymbol{S}$ with $N \cong N(\boldsymbol{S})$ iff (a) $(N,+)$ is Abelian, (b) $N$ has an identity 1 , (c) There is some $d \in N_{d}$ such that $N_{0}$ is generated by $\{1, d\}$.

Proof. Let $N$ be a near-ring with (a)-(c), we know that $N$ is isomorphic to a subnear-ring $\bar{N}$ of $M(N,+)$ [2]. 
Let $\bar{d}$ and $\overline{1}$ be the images of $d$ and 1 in $\bar{N}$. Since $d$ is distributive, $\bar{d}$ is an endomorphism of $(N,+)$ and $\overline{1}=i d_{N} \cdot \bar{N}_{0}$ is generated by id and $\bar{d}$, whence

$\bar{N}_{0}=\left\{z_{0} i d+z_{1} \bar{d}+\cdots+z_{n} \bar{d}^{n} \mid z_{i} \in Z\right\} \quad(n$ is non negative integer). Now let $(A,+):=(Q,+):=(N,+)$ and $F(q, a):=q \bar{d}+0 a$. Then $(Q, A, F)$ is $a$ linear GSA, Since $(N,+)$ is abelian. Since $\bar{d}=f_{0}$ we get $\bar{N}_{0}=N_{0}(\boldsymbol{S})$. Furthermore, take $f \in N_{c}(\boldsymbol{S})$. We get $f=0 f=0\left(\Sigma \pm f_{\alpha_{i}}\right)=\Sigma \pm\left(0 f_{\alpha_{i}}\right)$ with $0 f_{\alpha_{i}}=\bar{f}_{a_{1}} f_{0}^{n-1}+\cdots+\bar{f}_{a_{n}}=0 \bar{f}_{a_{1}} \bar{d}^{n-1}+\cdots+0 \bar{f}_{a_{n}} \in 0 \bar{N}=\bar{N}_{c}$. This shows $N_{c}(\boldsymbol{S}) \subseteq \bar{N}_{c}$. Conversely, every $\bar{c} \in \bar{N}_{c}$ (with constant value $c$ ) is in $N_{c}(\boldsymbol{S})$ since $\bar{c}=\bar{f}_{c}$. Hence $N(\boldsymbol{S})=\bar{N} \cong N$.

It is customary in algebraic automata theory to consider the semigroup-epimorphism $A^{*} \rightarrow N(\boldsymbol{S})$ given by $a \rightarrow f_{a}$. The idea of simultaneous inputs enables us to transfer this epimorphism from semigroups to nearrings. We can, for instance, interpret $a_{1} a_{2}+2 a_{2}$ as being the complex input "input sequence $a_{1} a_{2}$ together with the simultaneous input $a_{2}$ (in double strength)". We extend $A$ to the free near-ring $A^{\#}$ over $A$. If $a^{\#}=w\left(a_{1}, \cdots, a_{n}\right)$ is a word in $A^{\#}$ we define $f_{w\left(a_{1} \cdots a_{n}\right)}:=w\left(f_{a_{1}}, \cdots, f_{a_{n}}\right)$, and $F^{\#}\left(q, a^{\#}\right):=q f_{a^{\#}}$. Thus we get an extended simultaneous sequential GSA $\boldsymbol{S}^{\#}:=\left(Q, A^{\#}, F^{\#}\right)$. Let $I$ be $\left\{a^{\#} \in A^{\#} \mid f_{a^{\#}}\right.$ is the zero map\}. Then $I$ is $a$ near-ring ideal and we get by the homomorphism theorem: $A^{\#} / I \cong N\left(\boldsymbol{S}^{\#}\right)=N(\boldsymbol{S})$

If we had used right near-rings, we would have $N(\boldsymbol{S})$ anti-isomorphic to $A^{\#} / I$. Hence $N(\boldsymbol{S})$ can be viewed as a homomorphic image of $A^{\#}$. It is, however, impossible to give a nice canonical form for all elements of $A^{\#}$.

A possible relief comes from the observation that one might replace $A^{\#}$ by $A^{v}$, the free algebra in a variety $v$ of near-rings containing $N(\boldsymbol{S})$ (for instance, one might take $v$ as the variety generated by $N(\boldsymbol{S})$ ).

Attention! If $A$ already bears some additive structure, this new addition can (and in most cases will) be different from the given addition in $A$ ! In particular, our new addition is one in $A^{\#}$ and not in $A^{*}$.

In the linear case we saw that $N(\boldsymbol{S})$ is an affine nearring. Since the class of all affine near-rings is known to form a variety, it makes sense to look at free affine nearrings, the more so since we know how this monsters look like.

Let $\mathrm{A}$ be a set, $A^{*}$ the free monoid over $A$ and $\bar{A}$ the free affine near-ring over $A$. Then every element of $\bar{A}$ is a finite sum of elements $\pm \alpha_{i}$ with $\alpha_{i} \in(A \cup\{0\})^{*}$. In fact. Since $x(y+z)=x y+x z$, $(x+y) z=x z-x z 0+y z-y z 0+z 0$ and $(-x) y=-x y+y x 0+y 0$ are laws in the variety of affine near-rings, we can bring all expressions into \pm -sums of elements which are products of elements in $A \bigcup\{0\}$ (observe that we use left near-rings!)

Let $\boldsymbol{S}=(Q, A, F)$ be a GSA and $A^{\#}$ the free nearring on $A . \quad q_{1} \in Q$ is accessible from $q_{2} \in Q$ if there is some $\alpha \in A^{\#}$ with $q_{2} f_{\alpha}=q_{1} . S$ is accessible if each state $q$ is accessible from each other state. $N(\boldsymbol{S})$ is not only a near-ring, but it also operates on $Q$. obviously $Q$ is an $N(\boldsymbol{S})$ group via $q f_{a}$ in the usual meaning. $q_{1}$ is accessible from $q_{2}$ iff $q_{1} \in q_{2} N(\boldsymbol{S})$. Alternatively, $Q$ can be viewed as an $A^{\#}$-group via $q \alpha: q f_{\alpha}$. We have $\boldsymbol{S}$ is accessible iff $Q$ is an $N:=N(\boldsymbol{S})$-group with $0 N=Q$. In fact, if $\boldsymbol{S}$ is accessible then obviously $0 N=Q$. Conversely, suppose that $Q=0 N=0 N_{C}$. If $q \in Q$ then

$q N=q N_{0}+q N_{C}=q N_{0}+0 N_{C}=q N_{0}+Q=Q$, and $S$ is shown to be accessible.

It might be most useful to examine the relationship between generators, primitivity and accessibility more closely. Now we look at constructions of semiautomata and their corresponding syntactic near-rings.

Let $\boldsymbol{S}=(Q, A, F)$ and $\boldsymbol{S}^{\prime}=\left(Q^{\prime}, A, F^{\prime}\right)$ be GSA with identical input sets. A group homomorphism $h: Q \rightarrow Q^{\prime}$ is called a GSA-homomorphism if $h\left(q f_{a}\right)=h(q) f_{a}^{\prime}$ holds for all $q \in Q$ and $a \in A$ (with $f_{a}^{\prime}\left(q^{\prime}\right):=F^{\prime}\left(a, q^{\prime}\right)$ of course).

Theorem 3. Let $h: S \rightarrow \boldsymbol{S}^{\prime}$ be a GSA-epimorphism. Then there exists $a$ near-ring epimorphism $\bar{h}$ from $N(\boldsymbol{S})$ to $N\left(\boldsymbol{S}^{\prime}\right)$ with $h(q n)=h(q) \bar{h}(n)$ for all $q \in Q$ and $n \in N(\boldsymbol{S})$.

Proof. If $n \in N(\boldsymbol{S}), n$ is a word $n=w=w\left(f_{a_{1}}, \cdots, f_{a_{k}}\right)=f_{w\left(a_{1}, \cdots, a_{k}\right)}$ in $f_{a_{1}}, \cdots, f_{a_{k}}$. Then $h\left(q f_{w}\right)=h(q) f_{w}^{\prime}$ by induction on the length of $w$. Define $\bar{h}\left(f_{w}\right):=f_{w}^{\prime} \cdot \bar{h}$ is well-defined since $f_{w}=f_{w}^{\prime}$, implies $h(q) f_{w}^{\prime}=h\left(q f_{w}\right)=h\left(q f_{w^{\prime}}\right)=h(q) f_{w^{\prime}}^{\prime}$, for all $q \in Q$. Since $h$ is surjective, $f_{w}^{\prime}=f_{w^{\prime}}^{\prime}$ follows. Obviously, $\bar{h}$ is a near-ring epimorphism and $h(q n)=h\left(q f_{w}\right)=h(q) f_{w}^{\prime}=h(q) \bar{h}(n)$ is also true for all $q \in Q$ and $n \in N(\boldsymbol{S})$.

An automaton is a quintuple $\boldsymbol{A}=(Q, A, B, F, G)$, where $(Q, A, F)$ is a semiautomaton, $B$ a set (the output set) and $G: Q \times A \rightarrow B$ a function (called the output function of $\boldsymbol{A}$ ). If $Q$ is a group, $\boldsymbol{A}$ is called a groupautomaton (abbreviated by GA). We call $\boldsymbol{A}$ a homomorphic GA if $Q, A, B$ are groups and $F, G$ are homomorphisms. $\boldsymbol{A}$ is called a linear GA or linear automaton or linear sequential machine if $Q, A, B$ are $R$-modules for some ring $R$ and $F, G$ are $R$-linear maps [1].

In many cases, however, outputs do play an essential role. For instance, if one wants to connect two (or more) automata in series. For doing that, consider $\boldsymbol{A}=(Q, A, B, F, G)$ and $\boldsymbol{A}^{\prime}=\left(Q^{\prime}, B, C, F^{\prime}, G^{\prime}\right)$. The 


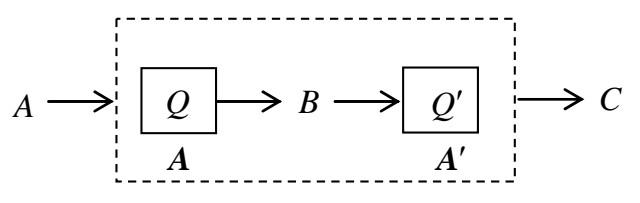

Series connection $A s A^{\prime}$

outputs of $\boldsymbol{A}$ shall be the inputs of $\boldsymbol{A}^{\prime}$

More formally, $\boldsymbol{A} s \boldsymbol{A}^{\prime}:=\left(Q \times Q^{\prime}, A, C, F^{\prime \prime}, G^{\prime \prime}\right)$ with $\left.F^{\prime \prime}\left(q, g^{\prime}\right), a\right):=\left(F(q, a), F^{\prime}\left(q^{\prime}, G(q, g)\right)\right.$ and

$G^{\prime \prime}\left(\left(q, q^{\prime}\right), a\right):=G^{\prime}\left(G(q, a), q^{\prime}\right)$.

If $\boldsymbol{A}$ and $\boldsymbol{A}^{\prime}$ are linear GA then $N\left(\boldsymbol{A} s \boldsymbol{A}^{\prime}\right)$ is the near-ring $N(\boldsymbol{A}) \quad s N\left(\boldsymbol{A}^{\prime}\right)$ additively generated by all pairs of the form $\left(f_{0}, f_{0}^{\prime}\right)^{k}$ ( $n$ is non negative integer), the constant-map-pairs $\left(\bar{f}_{a}, \bar{f}_{G(0, a)}\right)(a \in \boldsymbol{A})$ and all

$\left(0, k p_{0}\right)$ ( $n$ is non negative integer), with $p_{0}: Q \rightarrow M_{C}\left(Q^{\prime}\right), \quad q \rightarrow \bar{f}_{G(q, 0)}$.

Let $A^{*}$ and $B^{*}$ denote the free monoids over $A$ and $B$, respectively. For $q \in Q$ let $s_{q}: A^{*} \rightarrow B^{*}$ be defined by $s_{q}(\Lambda):=\Lambda, \quad s_{q}(a):=G(q, a)$,

$s_{q}\left(a_{1}, a_{2}\right):=G\left(q, a_{1}\right) G\left(F\left(q, a_{1}\right), a_{2}\right)$

$=s_{q}\left(a_{1}\right) s_{F\left(q, a_{1}\right)}\left(a_{2}\right)$ and proceed inductively with $s_{q}\left(a_{1} a_{2} \cdots a_{n}\right)=s_{q}\left(a_{1} a_{2} \cdots a_{n-1}\right) G\left(F\left(q, q_{1}, \cdots, q_{n-1}\right), a_{n}\right)$.

$s_{q}: A^{*} \rightarrow B^{*}$ is called the sequential (input-output-) function of $\boldsymbol{A}$ at $q$. If $\boldsymbol{A}$ is $a \mathrm{GA}, s_{0}=: s_{\boldsymbol{A}}$ is called the sequential function of $\boldsymbol{A}$. Furthermore, call $q, q^{\prime} \in Q$ equivalent states $\left(q \sim q^{\prime}\right)$ if $s_{q}=s_{q^{\prime}}$ (i.e. if $q$ and $q^{\prime}$ induce the same input-output-behaviour).

It might make sense to extend $s_{q}$ from $A^{\#}$ to $B^{\#}$, where $A^{\#}$ and $B^{\#}$ are the free near-rings [2] in $a$ variety which contains the one generated by $N(\boldsymbol{A})$ if we define

$$
s_{q}\left(a_{1}+a_{2}\right):=G\left(q, a_{1}\right)+G\left(q, a_{2}\right)=s_{q}\left(a_{1}\right)+s_{q}\left(a_{2}\right) .
$$

If $\boldsymbol{A}=(Q, A, B, F, G)$ is homomorphic we get for $q, q^{\prime}, q^{\prime \prime} \in Q$ :

If $q^{\prime} \sim q^{\prime \prime}$ then $s_{q^{\prime}}=s_{q^{\prime \prime}}$. Let $q \in Q$. Then

$$
\begin{aligned}
& S_{q+q^{\prime}}(\Lambda)=\Lambda=s_{q+q^{\prime \prime}}(\Lambda) ; \\
& S_{q+q^{\prime}}(a)=G\left(q+q^{\prime}, a\right)=G(q, a)+G\left(q^{\prime}, a\right)-G(0, a) \\
& =G(q, a))+G\left(q^{\prime \prime}, a\right)-G(0, a)=G\left(q+q^{\prime \prime}, a\right) \\
& =s_{q+q^{\prime \prime}}(a) \\
& s_{q+q^{\prime}}\left(a_{1} a_{2}\right) \\
& =s_{q+q^{\prime}}\left(a_{1}\right) G\left(\left(F\left(q, a_{1}\right), a_{2}\right)\right. \\
& \left.\quad+\left(F\left(q^{\prime}, a_{1}\right), a_{2}\right) \cdot\left(F\left(0, a_{1}\right), a_{2}\right)\right) \\
& =s_{q+q^{\prime \prime}}\left(a_{1}\right) G\left(F\left(q, a_{1}\right), a_{2}\right) \\
& \left.\left.\quad+F\left(q^{\prime \prime}, a_{1}\right), a_{2}\right)-\left(F\left(0, a_{1}\right), a_{2}\right)\right) \\
& =S_{q+q^{\prime \prime}}\left(a_{1} a_{2}\right)
\end{aligned}
$$

and so on, hence $s_{q+q^{\prime}}=s_{q+q^{\prime \prime}}$, whence $q+q^{\prime} \sim q+q^{\prime \prime}$.
Similarly, if $\quad q \sim q^{\prime} a \in A \quad$ and $\quad n=f_{a_{1} \cdots a_{k}} \in N(\boldsymbol{A})$ then

$$
\begin{aligned}
& s_{q n}(a) \\
& =G\left(q f_{a_{1} \cdots a_{k}}, a\right)=G\left(F\left(q, a_{1}, \cdots, a_{k}\right), a\right) \\
& =G\left(F\left(q^{\prime}, a_{1} \cdots a_{k}\right), a\right)=G\left(q^{\prime} f_{a_{1} \cdots a_{k}}, a\right)=s_{q^{\prime} n}(a)
\end{aligned}
$$

and induction shows $q n \sim q^{\prime} n$. We there fore get

Theorem 4. Let $\boldsymbol{A}$ be $a$ homomorphic GA. Then $\sim$ is a congruence relation in the $N(\boldsymbol{A})$-group $Q$. and $(a)$ $Q_{0}:=\{q \in Q \mid q \sim 0\} \quad$ is an ideal of ${ }_{N(A)} Q$; (b) $G(q, 0)=0$ for all $q \in Q_{0}$.

We might ask what $q \sim q^{\prime}$ means in detail

Theorem 5. Let $\boldsymbol{A}$ be homomorphic and $g_{0}: Q \rightarrow B, q \rightarrow q g_{0}=G(q, 0)$. Then $q \sim q^{\prime} \Leftrightarrow$ For any non negative integer $k, q\left(f_{0}^{k} g_{0}\right)=q^{\prime}\left(f_{0}^{k} g_{0}\right)$

Proof. Let $q \sim q^{\prime}$. We use induction on $k$ and start with $k=0$. If $a \in A$ then

$$
S_{q}(a)=G(q, a)=G(q, 0)+G(0, a)=q g_{0}+G(0, a) .
$$

Since $S_{q}(a)=S_{q^{\prime}}(a)$ we get $q g_{0}=q^{\prime} g_{0}$. Now suppose theorem 5 holds for all words $\alpha=a_{1} a_{2} \cdots a_{k-1} \in A^{*}$ of length $k-1=: t$. Then for all $a \in A$,

$S_{q}(\alpha a)=S_{q^{\prime}}(\alpha a)$, hence $G\left(q f_{\alpha}, a\right)=G\left(q^{\prime} f_{\alpha}, a\right)$, we have,

$$
\begin{aligned}
G\left(q f_{\alpha}, a\right) & =G\left(q f_{0}^{k}+\sum_{i=1}^{t} f_{a_{i}} f_{0}^{t-i}, a\right) \\
& =G\left(q f_{0}^{k}, 0\right)+\sum_{i=1}^{t} G\left(f_{a_{i}} f_{0}^{t-i}, 0\right)+G(0, a)
\end{aligned}
$$

Similarly,

$$
G\left(q^{\prime} f_{\alpha}, a\right)=G\left(q^{\prime} f_{0}^{k}, 0\right)+\sum_{i=1}^{t} G\left(f_{a_{i}} f_{0}^{t-i}, 0\right)+G(0, a),
$$

hence $G\left(q f_{0}^{k}, 0\right)=G\left(q^{\prime} f_{0}^{k}, 0\right)$ and we get $q f_{0}^{k} G_{0}=q^{\prime} f_{0}^{k} g_{0}$. The converse is shown similarly.

A GA $\boldsymbol{A}=(Q, A, B, F, G)$ is reduced if $\sim$ is the equality. If $\boldsymbol{A}$ is accessible (i.e. if ( $Q, A, F)$ is accessible) and reduced then $\boldsymbol{A}$ is called minimal [1]. Obviously, a homomorphic GA is reduced iff $G_{0}=\{0\}$, we have

Corollary 6. Let $\boldsymbol{A}=(Q, A, B, F, G)$ be a GA. Then (a) $\boldsymbol{A}_{a}:=\left(Q(N(\boldsymbol{A}))=: Q_{a}, A, B, F / Q_{a} \times A, G / Q_{a} \times A\right)$ is accessible; (b) $Q=0 N(A)$; (c) A/ : $=\left(Q / \sim, A, B, F_{\sim}, Q_{\sim}\right)$ with $F_{\sim}([q], a):=[F(q, a)]$ and $G_{\sim}([q], a):=G(q, a)$ is reduced; (d) $\boldsymbol{A}_{a} / \sim$ is minimal.

The proofs are straightforward. In looking for criteria to decide if a given GA $\boldsymbol{A}$ is minimal or not, we obviously have to view $Q$ not only as an $N(\boldsymbol{A})$-group but also have to care about $B$. 
Corollary 7. Let $\boldsymbol{A}$ be a homomorphic GA. Then $\boldsymbol{A}$ is reduced iff ${ }_{N(A)} Q$ has no non-zero ideals $p$ with $p g_{0}=\{0\}$.

Proof. If ${ }_{N(A)} Q$ has no such ideals then $Q_{0}=\{0\}$ and $\boldsymbol{A}$ is reduced. So suppose that conversely $\boldsymbol{A}$ is reduced and that $P<_{N(A)} Q$ has $G(P, 0)=p g_{0}=0$ for all $p \in P$. If $p \in P$, we see by similar arguments that $p \sim 0$, hence $p=0$, whence $P=\{0\}$.

From corollary 7 we get

Corollary 8. Let $\boldsymbol{A}$ be a homomorphic GA. Then $\boldsymbol{A}$ is minimal iff ${ }_{N(A)} Q$ is generated by 0 and does not contain non-zero ideals which are annihilated by $g_{0}$.

\section{REFERENCES}

[1] S. Eilenberg, "Automata, Language, and Machines," Academic Press, New York, 1974.
[2] G. Pilz, “Near Rings,” North-Holland, Amsterdam, 1977.

[3] S. F. You, M. Cao and Y. J. Feng, "Semiautomata and Near Rings," Quantitative Logic and Soft Computing, Vol. 5, 2012, pp. 428-431.

[4] S. F. You, H. Y. Zhao, Y. J. Feng and M. Cao, "An Application of Eulerian Graph to PI on Mn(C)," Applied Mathematics, Vol. 3, No. 7, 2012, pp. 809-811.

[5] S. F. You, "An Application of Eulerian Graph to Polynomial Identity," IEEE Proceedings of the 2011 International Conference on Computational Intelligence and Software Engineering (CiSE 2011), Wuhan, 9-11 December 2011.

[6] S. F. You, et al., "Eulerian Graph and Polynomial Identities on Matrix Rings,” Advances in Mathematics, Vol. 32, No. 4, 2003, pp. 425-428.

[7] S. F. You, "The Primitivity of Extended Centroid Extension on Prime GPI-Rings," Advances in Mathematics, Vol. 29, No. 4, 2000, pp. 331-336. 